\title{
Les canicules de l'été 2003 : un événement météorologique exceptionnel dans le quart nord- est de la France
}

The hotwawes of the summer 2003: An atypical weatherevents in North-Eastern France

Die Hitzewellen vom Sommer 2003 : ein aussergewöhnliches Wetterereigniss im Nord-Osten Frankreichs.

Laurent Wahl, Patrice Paul, Claude Pichard, Nicolas Mory, Gilles Drogue et Laurent Pfister

\section{OpenEdition}

Journals

Édition électronique

URL : http://journals.openedition.org/rge/362

DOI : $10.4000 /$ rge.362

ISSN : 2108-6478

Éditeur

Association des géographes de l'Est

Édition imprimée

Date de publication : 1 avril 2005

Pagination : 67-77

ISSN : 0035-3213

Référence électronique

Laurent Wahl, Patrice Paul, Claude Pichard, Nicolas Mory, Gilles Drogue et Laurent Pfister, « Les canicules de l'été 2003 : un événement météorologique exceptionnel dans le quart nord-est de la France », Revue Géographique de l'Est [En ligne], vol. 45 / 2 | 2005, mis en ligne le 10 juin 2009, consulté le 07 septembre 2020. URL : http://journals.openedition.org/rge/362 ; DOI : https://doi.org/10.4000/ rge.362

Ce document a été généré automatiquement le 7 septembre 2020

Tous droits réservés 


\section{Les canicules de l'été 2003 : un événement météorologique exceptionnel dans le quart nord-est de la France}

The hotwawes of the summer 2003: An atypical weatherevents in North-Eastern France

Die Hitzewellen vom Sommer 2003 : ein aussergewöhnliches Wetterereigniss im Nord-Osten Frankreichs.

Laurent Wahl, Patrice Paul, Claude Pichard, Nicolas Mory, Gilles Drogue et Laurent Pfister

\section{NOTE DE L'ÉDITEUR}

Nous remercions tout particulièrement les organismes et les personnes suivantes pour la fourniture des données climatiques indispensables à la réalisation de cet article :

- Météo-France pour la fourniture des normales climatiques de la période 1971-2000 via la climathèque du CREBS (Cellule de Recherche en Environnement et BiotechnologieS) ;

- l'Institut Royal Météorologique (IRM) de Belgique pour la fourniture des données climatiques des stations de Florennes et d'Uccle;

- le service météorologique de l'aéroport de Luxembourg via le CREBS (Cellule de Recherche en Environnement et Biotechnologies);

- le Deutscher Wetterdienst par le biais du site http : www.dwd.de ;

- Guy Blanchet pour la fourniture de données complémentaires concernant certains postes du Centre Est de la France.

Article reçu le 15 janvier 2005 ; accepté le 28 février 2005 


\section{Introduction}

1 Le quart Nord-Est de la France a connu pendant l'été 2003 des températures exceptionnelles, comme dans la majeure partie de l'Europe occidentale et centrale. Il s'agit en effet de l'été le plus chaud observé depuis le début des mesures météorologiques à la fin du $18^{\mathrm{e}}$ siècle. Il semblerait même qu'il faille remonter jusqu'à l'année 1473 ou 1540 pour reconnaître un été aussi chaud (Brugger, 1882 ; Pfister, 1999). En 1540, " on parlera de chaleur et de sécheresse tellement intenses que l'on passe à pied sec toutes les rivières de l'Est et que les moulins doivent s'arrêter faute d'eau. On moissonne en juin et les vendanges se font en août ». Le débit du Rhin est tellement bas durant l'été 1540 qu'il peut être franchi à pied. Cette description rappelle un peu ce qui s'est passé en 2003.

2 La durée d'insolation a été remarquablement élevée, alors que la pluviométrie, assez variable dans l'espace, se rapproche de celle des étés les moins arrosés de la seconde moitié du XXe siècle.

3 Les conséquences sur la vie sociale et économique de cet aléa météorologique ont été importantes et très variées. La chaleur intense du début d'août s'est traduite par une augmentation brutale de la mortalité de l'ordre de $+110 \%$ à Strasbourg durant la période du 5 au 15 août (Besançenot, 2003); toutefois, elle a été inférieure à la surmortalité observée en Ile-de-France (+134\%), dans le Grand-Lyon (+184\%) ou à Dijon (+179\%). Sur l'ensemble du territoire français on comptabilise près de 14860 décès liés à cet épisode de canicule (Pison, 2004). En plus, la pollution de l'air a dépassé les seuils d'information critiques pour l'ozone. Des records de nombre de jours pollués ont été battus dans la majeure partie du quart Nord-Est de la France. En agriculture, les rendements ont subi des baisses sensibles, notamment pour le fourrage et le maïs non irrigué, alors que la croissance des arbres dans les forêts a été fortement perturbée. Les vendanges de crémant ont été engagées avant la fin août. Les niveaux des cours d'eau vosgiens ont été très faibles, tandis que le débit du Rhin est passé sous la barre des 400 $\mathrm{m}^{3} / \mathrm{s}$ à la fin de l'été. Plusieurs arrêtés préfectoraux ont limité l'usage de l'eau.

4 Avant d'analyser les causes de cet événement climatique exceptionnel à l'aide de documents divers (radiosondages et cartes synoptiques), il convient de replacer cet été au niveau des longues séries de données dont nous pouvons disposer. Sur l'ensemble du grand quart Nord-Est de la France nous avons sélectionné 22 stations météorologiques de premier et second ordre dont les caractéristiques sont mentionnées dans le tableau 1.

Tableau 1 : Caractéristiques des principales stations prises en considération dans l'étude.

\begin{tabular}{|l|l|l|l|l|l|}
\hline Station & Département & Altitude & Latitude & Longitude & Site \\
\hline Auxerre & Yonne & $207 \mathrm{~m}$ & $47^{\circ} 48^{\prime} \mathrm{N}$ & $3^{\circ} 33^{\prime} \mathrm{E}$ & Plateau \\
\hline Bâle - Mulhouse & Haut-Rhin & $267 \mathrm{~m}$ & $47^{\circ} 36^{\prime} \mathrm{N}$ & $7^{\circ} 31^{\prime} \mathrm{E}$ & Plaine \\
\hline Belfort & Territoire de Belfort & $422 \mathrm{~m}$ & $47^{\circ} 38^{\prime} \mathrm{N}$ & $6^{\circ} 53^{\prime} \mathrm{E}$ & Plateau \\
\hline Besançon & Doubs & $307 \mathrm{~m}$ & $47^{\circ} 10^{\prime} \mathrm{N}$ & $6^{\circ} 00^{\prime} \mathrm{E}$ & Colline \\
\hline
\end{tabular}




\begin{tabular}{|c|c|c|c|c|c|}
\hline Charleville-Mézières & Ardennes & $149 \mathrm{~m}$ & $49^{\circ} 47^{\prime} \mathrm{N}$ & $4^{\circ} 38^{\prime} \mathrm{E}$ & Vallée \\
\hline Colmar - Meyenheim & Haut-Rhin & $209 \mathrm{~m}$ & $47^{\circ} 55^{\prime} \mathrm{N}$ & $7^{\circ} 24^{\prime} \mathrm{E}$ & Plaine \\
\hline Danne-et-Quatre-Vents & Moselle & $377 \mathrm{~m}$ & $48^{\circ} 46^{\prime} \mathrm{N}$ & $7^{\circ} 18^{\prime} \mathrm{E}$ & Plateau \\
\hline Dijon - Longvic & Côte-d'Or & $222 \mathrm{~m}$ & $47^{\circ} 16^{\prime} \mathrm{N}$ & $5^{\circ} 05^{\prime} \mathrm{E}$ & Plaine \\
\hline Épinal - Dogneville & Vosges & $317 \mathrm{~m}$ & $48^{\circ} 13^{\prime} \mathrm{N}$ & $6^{\circ} 27^{\prime} \mathrm{E}$ & Vallée \\
\hline Langres & Haute-Marne & $467 \mathrm{~m}$ & $47^{\circ} 50^{\prime} \mathrm{N}$ & $5^{\circ} 19^{\prime} \mathrm{E}$ & Plateau \\
\hline Lons-le-Saunier & Jura & $243 \mathrm{~m}$ & $46^{\circ} 40^{\prime} \mathrm{N}$ & $5^{\circ} 32^{\prime} \mathrm{E}$ & Vallée \\
\hline Loxéville - Erneville & Meuse & $313 \mathrm{~m}$ & $48^{\circ} 44^{\prime} \mathrm{N}$ & $5^{\circ} 23^{\prime} \mathrm{E}$ & Plateau \\
\hline Luxeuil & Haute-Saône & $278 \mathrm{~m}$ & $47^{\circ} 47^{\prime} \mathrm{N}$ & $6^{\circ} 21^{\prime} \mathrm{E}$ & Vallée \\
\hline Mâcon & Saône-et-Loire & $216 \mathrm{~m}$ & $46^{\circ} 18^{\prime} \mathrm{N}$ & $4^{\circ} 48^{\prime} \mathrm{E}$ & Coteau \\
\hline Metz - Augny & Moselle & $190 \mathrm{~m}$ & $49^{\circ} 05^{\prime} \mathrm{N}$ & $6^{\circ} 08^{\circ} \mathrm{E}$ & Plateau \\
\hline Nancy - Essey & Meurthe-et-Moselle & $212 \mathrm{~m}$ & $48^{\circ} 41^{\prime} \mathrm{N}$ & $6^{\circ} 13^{\prime} \mathrm{E}$ & Vallée \\
\hline Nancy - Ochey & Meurthe-et-Moselle & $336 \mathrm{~m}$ & $48^{\circ} 35^{\prime} \mathrm{N}$ & $5^{\circ} 58^{\prime} \mathrm{E}$ & Plateau \\
\hline Nevers & Nièvre & $180 \mathrm{~m}$ & $47^{\circ} 00^{\prime} \mathrm{N}$ & $3^{\circ} 06^{\prime} \mathrm{E}$ & Vallée \\
\hline Saint-Dizier & Haute-Marne & $139 \mathrm{~m}$ & $48^{\circ} 38^{\prime} \mathrm{N}$ & $4^{\circ} 54^{\prime} \mathrm{E}$ & Vallée/coteau \\
\hline Strasbourg - Entzheim & Bas-Rhin & $151 \mathrm{~m}$ & $48^{\circ} 33^{\prime} \mathrm{N}$ & $7^{\circ} 38^{\prime} \mathrm{E}$ & Plaine \\
\hline Reims & Marne & $91 \mathrm{~m}$ & $49^{\circ} 18^{\prime} \mathrm{N}$ & $4^{\circ} 02^{\prime} \mathrm{E}$ & Plaine \\
\hline Troyes - Barberey & Aube & $118 \mathrm{~m}$ & $48^{\circ} 20^{\prime} \mathrm{N}$ & $4^{\circ} 01^{\prime} \mathrm{E}$ & Vallée \\
\hline
\end{tabular}

5 D'autres stations périphériques de la zone d'étude ont également été prises en considération (figure 1). Elles sont au nombre de 14 (Bourges, Clermont-Ferrand, Florennes, Genève, Karlsruhe, Lille - Lesquin, Luxembourg - Findel, Lyon - Bron, Melun, Neuchâtel, Saarbrücken, Saint-Quentin, Trier - Petrisberg, Uccle). Plus de la moitié d'entre-elles se situent à l'étranger (1 au Luxembourg, 2 en Belgique, 3 en Allemagne, 2 en Suisse).

\section{La position de l'été 2003}

6 Pour montrer le caractère exceptionnel de l'été 2003, il convient de le replacer par rapport aux normales climatiques de divers paramètres climatiques (température moyenne, températures minimales et maximales, seuils de températures, 
précipitations, insolation). Dans cette analyse, nous nous limiterons aux mois de juin, juillet et août qui représentent l'été météorologique qui va du $1^{\mathrm{er}}$ juin au 31 août.

\section{A. Les températures}

7 La rigueur d'un hiver ou d'un été ne peut s'apprécier que par les écarts observés par rapport à une normale climatique servant de référence. Cette dernière est établie à partir de 30 années d'observations. La plupart des écarts calculés se rapportent à la normale 1971-2000. Le nombre de jours de chaleur (température maximale supérieure ou égale à $+25^{\circ} \mathrm{C}$ ) ou de forte chaleur (température maximale supérieure ou égale à $+30^{\circ} \mathrm{C}$ ) constituent de bons indices pour montrer le caractère plus ou moins important de la chaleur d'un été.

\section{Les températures moyennes}

8 Si l'on analyse la moyenne des températures établie pour les trois mois d'été (juin, juillet et août), on remarque que l'été 2003 se caractérise par des anomalies thermiques positives remarquables qui s'échelonnent entre $+2,5{ }^{\circ} \mathrm{C}$ (Charleville-Mézières) et $+5,3^{\circ} \mathrm{C}$ (Lons-le-Saunier). L'excédent thermique moyen se situe aux alentours de $4{ }^{\circ} \mathrm{C}$ pour l'ensemble des postes du secteur géographique pris en considération (zone en grisé de la figure 1). Cette valeur est particulièrement élevée en comparaison des étés les plus chauds de l'après-guerre (1947, 1976, 1983 et 1994) où l'on note des écarts de $+1,5^{\circ} \mathrm{C}$ à $+2,5^{\circ} \mathrm{C}$.

Figure 1 : Moyenne des températures minimales observées au cours des 3 mois de l'été 2003.

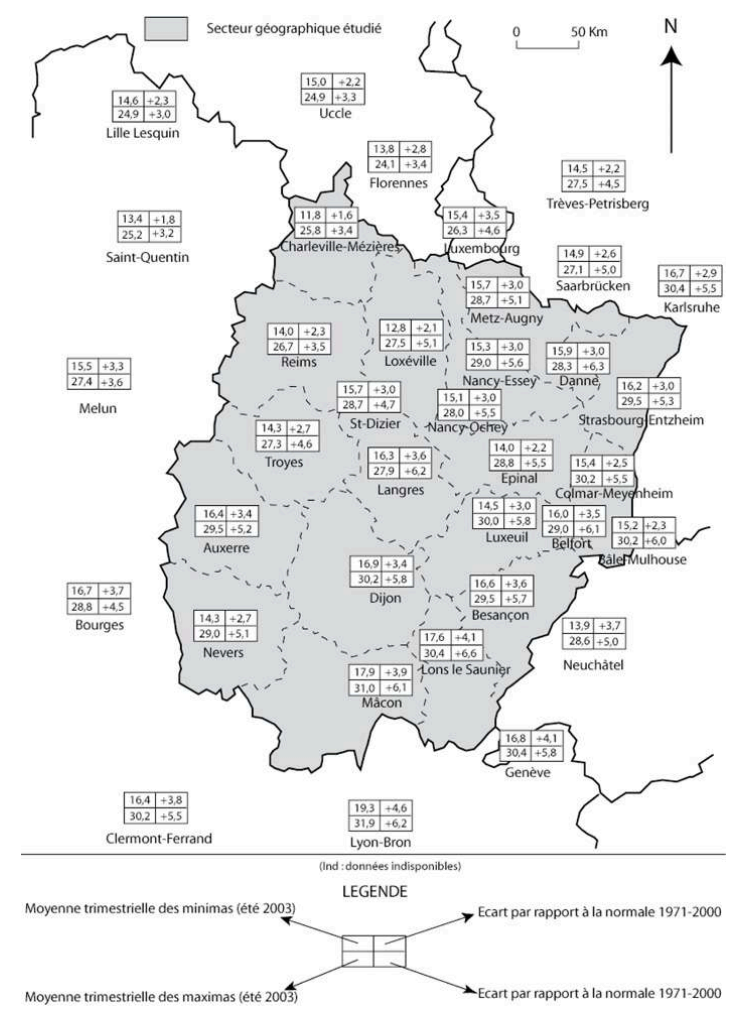


9 La figure 2 et le tableau 2 montrent qu'à Nancy-Essey, avec un excédent de $+4,6^{\circ} \mathrm{C}$ par rapport à la moyenne considérée, l'été 2003 devance nettement les étés 1983, 1994 et 1947 qui arrivent respectivement en seconde, troisième et quatrième position.

Figure 2 : Températures moyennes observées à Nancy-Essey durant les étés de la période 1945-2003.

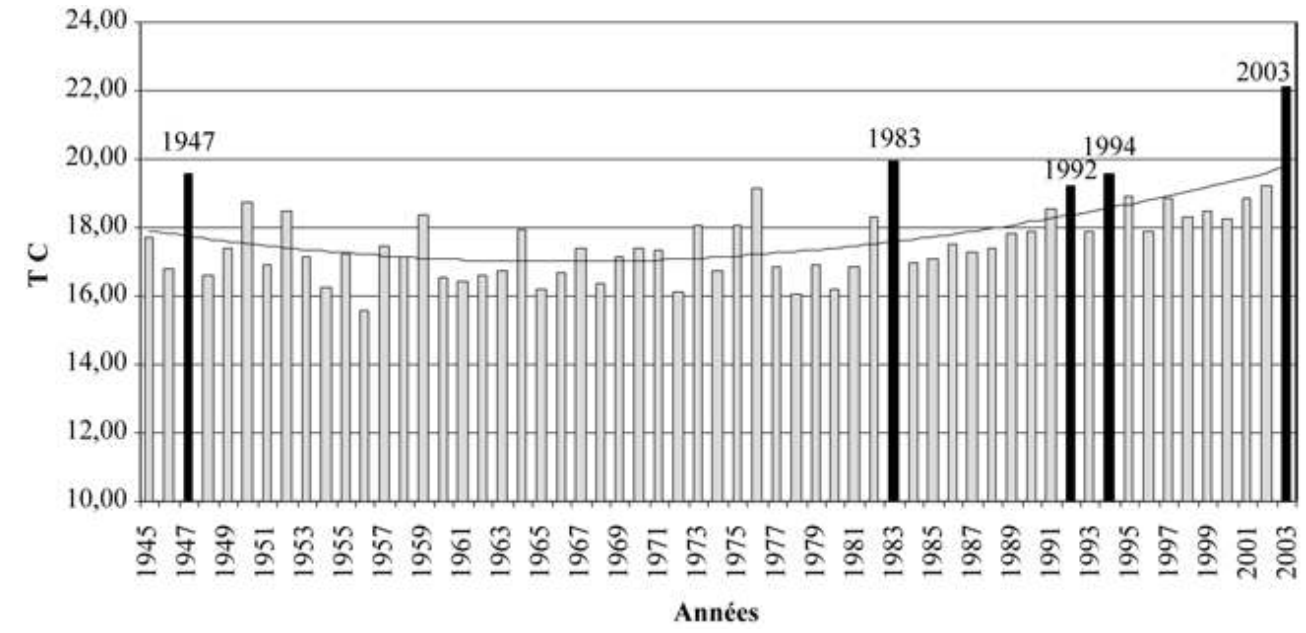

Tableau 2 : Écarts thermiques à la moyenne établie de 1945 à 2003 pour la station de Nancy-Essey.

\begin{tabular}{|l|l|l|l|l|l|}
\hline Été & $T^{\circ} \mathrm{C}$ moy & Juin & Juillet & Août & Écart/Été \\
\hline $\mathbf{2 0 0 3}$ & $+22,15$ & $+5,4$ & $+2,6$ & $+5,6$ & $+4,59$ \\
\hline $\mathbf{1 9 8 3}$ & $+19,93$ & $+1,1$ & $+3,9$ & $+1,9$ & $+2,29$ \\
\hline $\mathbf{1 9 9 4}$ & $+19,60$ & $+0,9$ & $+3,9$ & $+1,1$ & $+1,96$ \\
\hline $\mathbf{1 9 4 7}$ & $+19,57$ & $+1,8$ & $+1,8$ & $+2,2$ & $+1,93$ \\
\hline $\mathbf{1 9 9 2}$ & $+19,23$ & $+0,9$ & $+1,4$ & $+2,5$ & $+1,59$ \\
\hline $\mathbf{2 0 0 2}$ & $+19,22$ & $+3,0$ & $+0,6$ & $+2,5$ & $+1,58$ \\
\hline \hline $\mathbf{1 9 7 6}$ & $+19,17$ & $+2,6$ & $+2,4$ & $-0,4$ & $+1,53$ \\
\hline
\end{tabular}

10 Un constat similaire peut être établi pour les mois de juin et d'août avec des écarts de $+5,4{ }^{\circ} \mathrm{C}$ et $+5,6^{\circ} \mathrm{C}$ ! Le mois de juillet enregistre un excédent plus modéré de $+2,6{ }^{\circ} \mathrm{C}$; pour ce mois des écarts nettement supérieurs ont été observés en 1983 et 1994 avec $+3,9^{\circ} \mathrm{C}$. Ce sont donc les mois de juin et d'août qui sont le plus à l'origine du caractère exceptionnel de l'été 2003. 


\section{Les températures minimales et maximales mensuelles (figure 1)}

11 L'anomalie thermique est plus marquée pour les températures maximales $\left(+3,4^{\circ} \mathrm{C}\right.$ à $\left.+6,6^{\circ} \mathrm{C}\right)$ que pour les températures minimales $\left(+1,6^{\circ} \mathrm{C} \grave{\mathrm{a}}+4,1^{\circ} \mathrm{C}\right)$ en raison d'une forte insolation diurne et de nuits claires, favorisant la baisse des températures.

Il est intéressant de relever que les excédents ne sont pas de la même ampleur pour les 3 mois d'été : juin se traduit par des excédents un peu plus marqués qu'en août pour l'ensemble des postes avec des écarts remarquables par rapport à la normale qui s'échelonnent entre $+2,7{ }^{\circ} \mathrm{C}$ et $+6,8^{\circ} \mathrm{C}$, souvent supérieurs à ceux d'août $\left(+3,0^{\circ} \mathrm{C}\right.$ à $\left.+6,2^{\circ} \mathrm{C}\right)$, tandis qu'en juillet, les excédents thermiques ont été plus modestes $\left(+0,7^{\circ} \mathrm{C}\right.$ à $\left.+2,5^{\circ} \mathrm{C}\right)$.

\section{Les journées de chaleur et de forte chaleur (figure 3)}

Figure 3 : Nombre de journées de forte chaleur. Records absolus de température enregistrés au cours des 3 mois de l'été 2003.

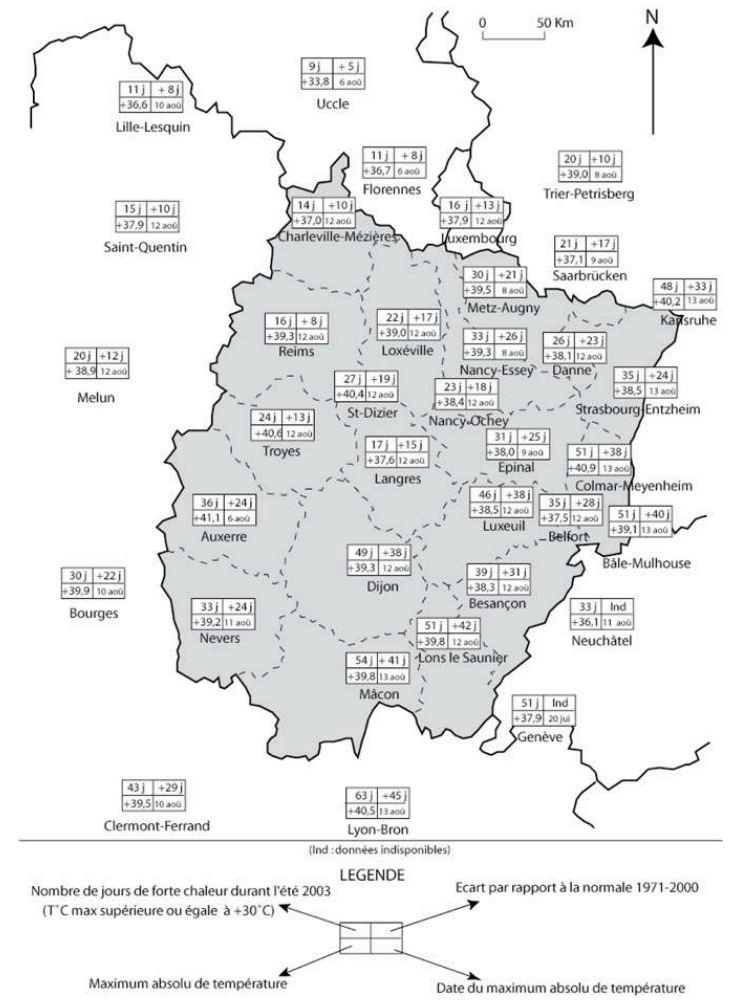

Trois vagues de chaleur de durée inégale se sont produites au cours de l'été 2003, ce qui a eu pour conséquence d'augmenter considérablement le nombre de jours de chaleur (température maximale supérieure ou égale à $+25^{\circ} \mathrm{C}$ ) qui a atteint souvent le double de la normale! Les valeurs s'échelonnent entre 52 jours à Charleville-Mézières (normale 1971-2000: 28 jours) et 81 jours à Colmar-Meyenheim (normale 1971-2000: 44 jours), dont 30 jours consécutifs du 28 mai au 30 juin 2003. Pour Nancy-Essey, on recense 77 jours qui représentent $85 \%$ du maximum théorique possible (92 jours). Des valeurs plus élevées sont observées dans le Fossé Rhénan où elles approchent $90 \%$. Pour l'ensemble des postes ce rapport est supérieur à $80 \%$ mis à part quelques stations situées au NordOuest d'une ligne Auxerre-Nancy-Strasbourg. 

$+30^{\circ} \mathrm{C}$ ) s'échelonne entre 14 à Charleville-Mézières et 54 jours à Mâcon; ces valeurs représentent parfois plus de 4 fois la normale (figure 3). Pour le mois d'août, on dénombre 16 jours en moyenne avec des valeurs comprises entre 12 et 24 jours pour ces mêmes postes. au 14 juin, 22 au 24 juin) qui ont plutôt affecté les secteurs situés au sud-est d'une ligne Saint-Dizier-Metz. Après un début de juillet plutôt maussade, une nouvelle vague de canicule apparaît du 15 au 20 juillet : de nombreux postes ont enregistré des pointes à $35^{\circ} \mathrm{C}$. Cela n'est rien en comparaison de la dernière vague de canicule qui se produit du 4 août au 13 août 2003 avec un caractère exceptionnel par sa durée et son intensité. On comptabilise dans la plupart des postes une série de 10 à 11 jours consécutifs où la température maximale a dépassé $35^{\circ} \mathrm{C}$, épisode sans précédent, ce seuil n'étant dépassé en moyenne qu'une seule fois au cours de l'été. Il arrive même fréquemment que le seuil des $35^{\circ} \mathrm{C}$ ne soit jamais atteint durant 2 ou 3 étés consécutifs. Durant cette période de nombreux records historiques de chaleur ont été balayés !

\section{Les records absolus de température $\left(\mathrm{T}^{\circ} \mathrm{C}\right.$ min et $\mathrm{T}^{\circ} \mathrm{C}$ max)}

records absolus ont le plus souvent moins d'effets sur la santé et la végétation que la durée d'une longue période de forte chaleur. La durée et l'intensité exceptionnelle de la vague de chaleur de la première quinzaine d'août peuvent être facilement identifiées par l'occurrence des jours torrides, caractérisés par des maxima supérieurs ou égaux à $+35^{\circ} \mathrm{C}$.

Des valeurs record ont été observées par toutes les stations, surtout pendant la première quinzaine d'août. Dès le 20 juillet, Meyenheim a enregistré $+38,2^{\circ} \mathrm{C}$, record absolu depuis l'ouverture de la station en 1957. Cette valeur remarquable a été largement battue le 13 août avec $+40,9^{\circ} \mathrm{C}$. Le seuil record de $40^{\circ} \mathrm{C}$ a aussi été dépassé aux stations météorologiques d'Auxerre $\left(+41,1^{\circ} \mathrm{C}\right)$, de Troyes $\left(+40,6^{\circ} \mathrm{C}\right)$ et de Saint-Dizier $\left(+40,4^{\circ} \mathrm{C}\right)$. A Auxerre on a comptabilisé 8 jours consécutifs avec des températures maximales dépassant $40^{\circ} \mathrm{C}$ ! Le seuil de $+39,5^{\circ} \mathrm{C}$ n'avait jamais été atteint à Strasbourg-Ville, qui dispose d'une très longue série de mesures remontant au début du $19^{\mathrm{e}}$ siècle. Il en est de même pour Nancy-Essey avec $+39,3^{\circ} \mathrm{C}$, valeur dépassant nettement l'ancien record de $+37,8^{\circ} \mathrm{C}$ enregistré en août 1998 . Sur les Vosges, massif dépourvu de longues séries de données climatiques, les maxima de $+30,2{ }^{\circ} \mathrm{C}$, relevés les 10 et 13 août au Markstein $(1180 \mathrm{~m})$, constituent des valeurs remarquables, qui sont probablement sans équivalent à cette altitude.

Une des particularités de cet été est que les records de chaleur ne se sont pas produits à la même date (figure 3) compte tenu de conditions climatiques locales fort différentes d'une région et d'un poste à l'autre (poste situé en zone urbanisée, en zone rurale).

Cette période caniculaire s'est accompagnée de températures minimales extrêmement élevées. Les minima supérieurs à $20^{\circ} \mathrm{C}$, appelés parfois "nuits tropicales", ont été fréquents, surtout en milieu urbain. Le minimum le plus élevé, observé à Nancy-Essey, a été de $+22,2{ }^{\circ} \mathrm{C}$ le 14 août. Des valeurs remarquables nettement plus élevées sont apparues sur les versants ou plateaux dégagés entre 400 et $600 \mathrm{~m}$ d'altitude, c'est-à-dire au-dessus de l'inversion de rayonnement nocturne, toujours présente par ciel clair et vent faible dans les vallées et les fossés tectoniques d'effondrement (Fossé rhénan, 
plaine de la Saône). Ainsi, un minimum de $+24,4^{\circ} \mathrm{C}$ a été mesuré à Nancy-Ochey (336 $\left.\mathrm{m}\right)$ et de $+25,9^{\circ} \mathrm{C}$ à Wangenbourg $(465 \mathrm{~m})$. On relève respectivement un maximum de 18 , 13, et 11 nuits tropicales à Mâcon, Dijon et à Danne-et-Quatre-Vents sur le plateau lorrain. On remarque que l'Alsace et la Lorraine ont été moins affectées par les nuits tropicales que la plaine de la Saône, la région parisienne et lyonnaise et la Côte d'Azur, où des minima jusqu'à $+28^{\circ} \mathrm{C}$ ont été relevés.

\section{B. L'insolation}

\section{Des durées annuelles d'insolation record}

L'insolation en 2003 a été exceptionnelle avec des cumuls annuels jamais égalés depuis le début des mesures. Pour l'ensemble des postes pris en considération les valeurs dépassent le seuil des 2000 heures, y compris à Charleville-Mézières (2 007 heures), dont l'ensoleillement moyen se situe autour de 1400 heures. Les cumuls s'échelonnent entre 2007 à Charleville et 2363 heures à Lons-le-Saunier, soit un excédent moyen de 525 heures! La plupart des records anciens remontent à l'année 1959, qui est restée célèbre pour la qualité des vins produits dans les vignobles de l'est de la France.

Tableau 3 : Nombre de jours de température minimale supérieure à $20^{\circ} \mathrm{C}$.

\begin{tabular}{|l|l|l|l|l|l|l|l|}
\hline Stations & Auxerre & Charleville & Colmar & Danne & Dijon & Loxéville & Luxeuil \\
\hline $\begin{array}{l}\text { Nombre de } \\
\text { jours }\end{array}$ & 10 jours & 0 jour & 1 jour & 11 jours & 13 jours & 1 jour & 0 jours \\
\hline Stations & Mâcon & $\begin{array}{l}\text { Nancy- } \\
\text { Essey }\end{array}$ & $\begin{array}{l}\text { Nancy- } \\
\text { Ochey }\end{array}$ & $\begin{array}{l}\text { Metz- } \\
\text { Augny }\end{array}$ & Saarbrücken & $\begin{array}{l}\text { Saint- } \\
\text { Dizier }\end{array}$ & Strasbourg \\
\hline $\begin{array}{l}\text { Nombre de } \\
\text { jours }\end{array}$ & 18 jours & 3 jours & 4 jours & 4 jours & 4 jours & 5 jours & 7 jours \\
\hline
\end{tabular}

Figure 4 : Cumuls annuels d'insolation à Nancy-Essey depuis 1930.

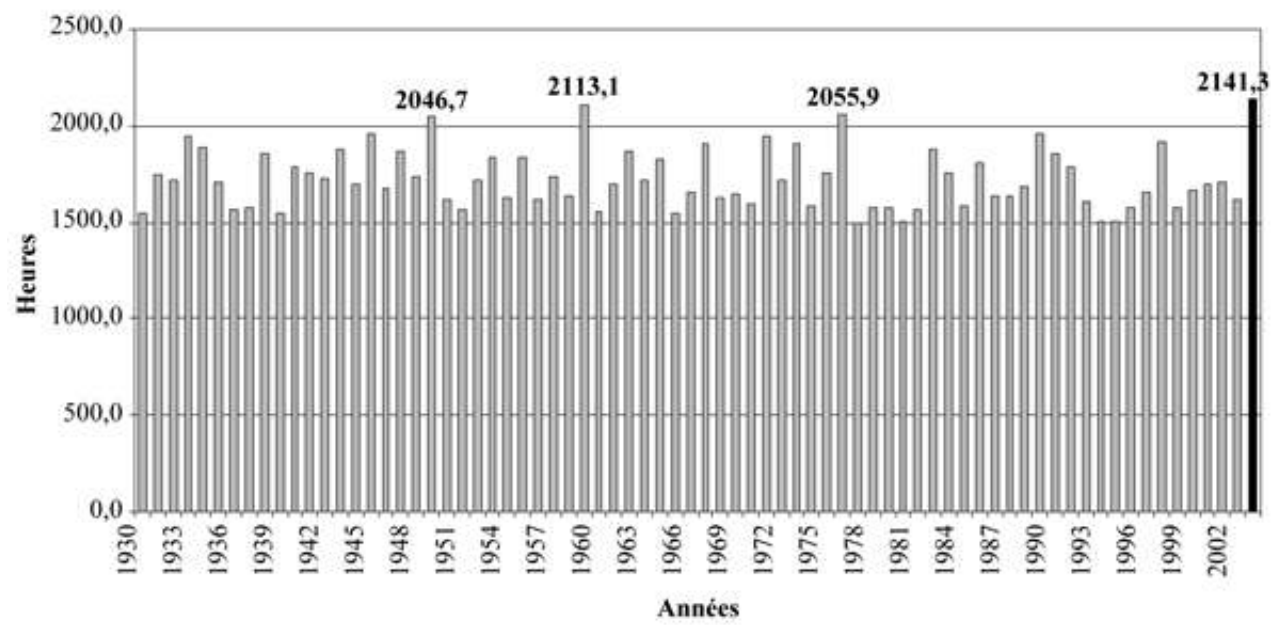

Revue Géographique de l'Est, vol. 45 / 2 | 2005 
21 La figure 4 fait apparaître nettement que l'année 2003 a été la plus ensoleillée depuis le début de la série d'observations en 1930. Entzheim, dont la série de mesures remonte à 1950, a également battu son record d'insolation avec 2197 heures.

\section{Des durées d'insolation estivales très contrastées et largement excédentaires}

Si l'on considère les cumuls d'insolation pour les 3 mois d'été, les valeurs divergent d'une station à l'autre avec des valeurs comprises entre 774 heures à Charleville et 934 heures à Lons-le-Saunier (figure 5). L'excédent le plus marqué est de $135 \%$ à la station de Bâle-Mulhouse.

Figure 5 : Totaux pluviométriques et cumul d'insolation observés au cours des 3 mois de l'été 2003 (juin, juillet, août).

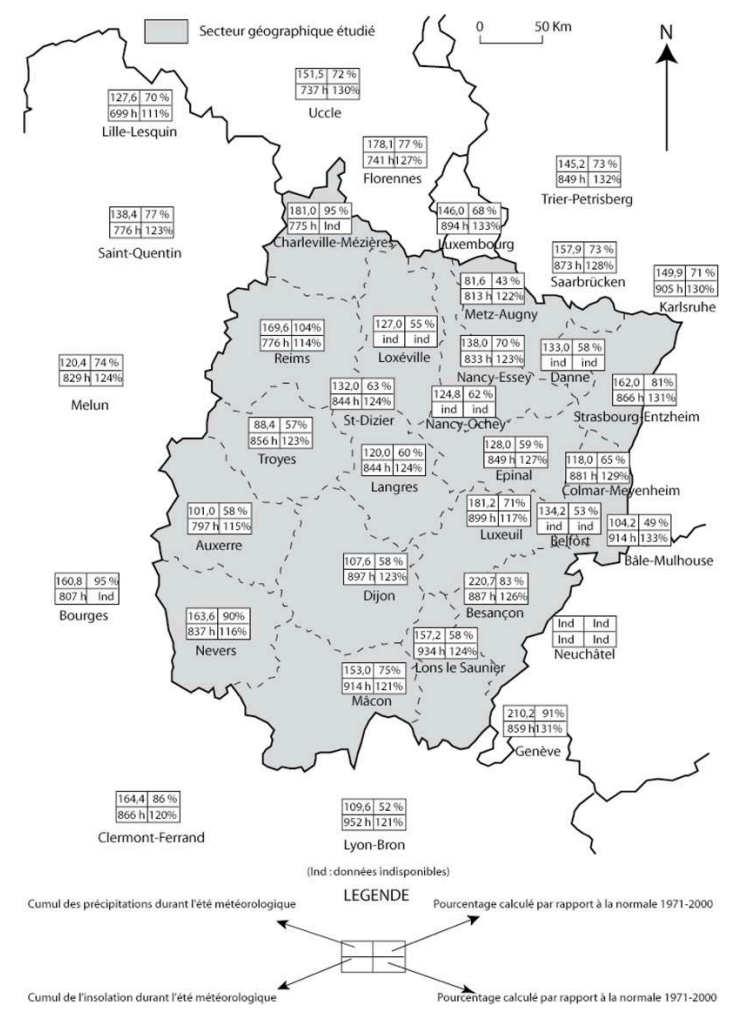

Si l'on compare ces valeurs estivales sur l'ensemble des séries disponibles, les résultats sont fort contrastés. À Strasbourg-Entzheim, l'été 2003 arrive largement en tête avec 865,2 heures contre 817,0 heures en 1976. A Nancy-Essey ce n'est pas le cas, étant donné que 1976 demeure au premier rang avec 896 heures, contre seulement 832 heures en 2003 (tableau 4). L'insolation de l'été 2003 n'arrive qu'en $5^{\mathrm{e}}$ position à Nancy-Essey. Seule l'insolation du mois d'août arrive en tête avec 315 heures. Cette variabilité spatiale est à mettre en relation avec le développement local de nuages d'instabilité qui peuvent masquer temporairement le soleil.

Tableau 4 : Les 5 valeurs maximales des durées d'insolation mensuelles et estivales observées à Nancy-Essey depuis 1930.

\begin{tabular}{|c|c|c|c|c|}
\hline Rang & Juin & Juillet & Août & ÉTÉ \\
\hline
\end{tabular}




\begin{tabular}{|l||l|l|l|l|}
\hline 1 & 358 (année 1976) & 340 (année 1983) & 315 (année 2003) & $\mathbf{8 9 6}$ (année 1976) \\
\hline 2 & 306 (année 2000) & 336 (année 1949) & 308 (année 1991) & $\mathbf{8 9 4}$ (année 1949) \\
\hline 3 & 305 (année 1962) & 332 (année 1971) & 291 (année 1962) & $\mathbf{8 4 8}$ (année 1983) \\
\hline 4 & 304 (année 1964) & 318 (année 1964) & 288 (année 1932) & $\mathbf{8 3 9}$ (année 1964) \\
\hline 5 & 298 (année 1949) & 313 (année 1967) & 285 (année 1976) & $\mathbf{8 3 2}$ (année 2003) \\
\hline
\end{tabular}

24 En Alsace, il convient de souligner qu'il n'y a eu aucun jour d'insolation nulle du 27 mai au 6 octobre, soit 133 jours consécutifs, ce qui est sans précédent pour une aussi longue période. À Nancy-Essey, cette durée est de 140 jours (du 20 mai au 6 octobre).

\section{Les précipitations}

L'année 2003 s'est caractérisée par un déficit pluviométrique plus ou moins intense qui a débuté dès le mois de février pour s'achever courant octobre. Toutefois cette sécheresse n'est pas aussi intense qu'en 1976, si l'on s'en tient strictement aux hauteurs de précipitations mesurées.

Les précipitations ont été généralement assez faibles, mais leur répartition dans l'espace géographique s'avère très inégale, surtout au mois de juin, caractérisé par de nombreux orages qui ont fourni des précipitations localement abondantes. Ainsi, les cumuls de précipitations de juin s'échelonnent de 15,2 $\mathrm{mm}$ à Saint-Dizier à 100,2 mm pour Besançon. L'ensemble des valeurs observées présente un déficit variant entre 20 et $80 \%$ à l'exception des stations de Strasbourg-Entzheim et de Besançon où elles sont conformes à la normale. Les précipitations de juillet ont été un peu plus abondantes avec des valeurs comprises entre $26,2 \mathrm{~mm}$ pour Troyes (déficit de $50 \%$ ) et 119,8 $\mathrm{mm}$ pour Luxeuil ( $141 \%$ de la normale); toutefois, elles restent généralement inférieures aux normales. Elles se sont produites sous la forme d'averses orageuses. Le mois d'août est particulièrement sec, avec des cumuls de pluies variant entre $16,4 \mathrm{~mm}$ (Epinal) et 73,0 mm (Mâcon), qui ne constituent toutefois pas des records de faible pluviométrie. Ces valeurs représentent 30 à $90 \%$ des précipitations attendues en août.

27 Au total, le déficit pluviométrique de l'été se situe entre 20 et près de $55 \%$ par rapport à un été normal. Les valeurs cumulées pour les 3 mois d'été varient dans une fourchette allant de 81,6 mm à Metz-Augny à 220,7 mm à Besançon. Néanmoins, on a dénombré au $20^{\mathrm{e}}$ siècle des étés beaucoup plus secs, comme en 1921, 1964, 1976 et 1983 (figure 5).

L'impression de sécheresse accrue réside dans le fait que de nombreux végétaux ont subi durablement une situation de stress hydrique qui a occasionné un jaunissement prématuré de leur canopée et une perte de leur feuillage. L'évapotranspiration potentielle (ETP) a été exceptionnellement élevée.

Tableau 5 : Précipitations enregistrées à Nancy durant les étés les plus secs.

\begin{tabular}{|l|l|l|l|l|l|}
\hline Années & Juin & Juillet & Août & Été & Total/Année \\
\hline 2003 & $53,2 \mathrm{~mm}$ & $37,4 \mathrm{~mm}$ & $46,8 \mathrm{~mm}$ & $137,4 \mathrm{~mm}$ & $567,6 \mathrm{~mm}$ \\
\hline
\end{tabular}




\begin{tabular}{|l|l|l|l|l|l|}
\hline 1983 & $38,1 \mathrm{~mm}$ & $25,4 \mathrm{~mm}$ & $10,1 \mathrm{~mm}$ & $73,6 \mathrm{~mm}$ & $805,4 \mathrm{~mm}$ \\
\hline 1976 & $34,3 \mathrm{~mm}$ & $66,7 \mathrm{~mm}$ & $3,8 \mathrm{~mm}$ & $104,8 \mathrm{~mm}$ & $536,0 \mathrm{~mm}$ \\
\hline 1964 & $24,3 \mathrm{~mm}$ & $22,9 \mathrm{~mm}$ & $56,2 \mathrm{~mm}$ & $103,4 \mathrm{~mm}$ & $469,0 \mathrm{~mm}$ \\
\hline Records & $\mathbf{8 , 3} \mathbf{~ m m}$ & $\mathbf{8 , 0} \mathbf{~ m m}$ & $\mathbf{3 , 8} \mathbf{~ m m}$ & $\mathbf{7 3 , 6} \mathbf{~ m m}$ & $\mathbf{4 6 9 , 0 ~ \mathbf { ~ m }}$ \\
\hline \hline Année & 1962 & 1979 & 1976 & 1983 & 1964 \\
\hline
\end{tabular}

Tableau 6 : Déficit hydrique observé au cours des 3 mois d'été de l'année 2003.

\begin{tabular}{|l|l|l|l|l|}
\hline Stations & Juin & Juillet & Août & Été 2003 \\
\hline \hline Besançon & $-69,5 \mathrm{~mm}$ & $-75,7 \mathrm{~mm}$ & $-107,3 \mathrm{~mm}$ & $-\mathbf{2 5 2 , 5} \mathbf{~ m m}$ \\
\hline \hline Colmar-Meyenheim & $-129,3 \mathrm{~mm}$ & $-109,6 \mathrm{~mm}$ & $-134,2 \mathrm{~mm}$ & $-\mathbf{3 7 3 , 1} \mathbf{~ m m}$ \\
\hline Lons-le-Saunier & $-149,2 \mathrm{~mm}$ & $-96,8 \mathrm{~mm}$ & $-92,2 \mathrm{~mm}$ & $-\mathbf{3 3 8 , 2} \mathbf{~ m m}$ \\
\hline Luxeuil & $-140,0 \mathrm{~mm}$ & $-19,4 \mathrm{~mm}$ & $-103,7 \mathrm{~mm}$ & $-\mathbf{2 6 3 , 1} \mathbf{~ m m}$ \\
\hline \hline Nancy-Essey & $-105,5 \mathrm{~mm}$ & $-122,2 \mathrm{~mm}$ & $-108,1 \mathrm{~mm}$ & $-\mathbf{3 3 5 , 8} \mathbf{~ m m}$ \\
\hline \hline Strasbourg-Entzheim & $-91,2 \mathrm{~mm}$ & $-89,1 \mathrm{~mm}$ & $-131,7 \mathrm{~mm}$ & $-\mathbf{3 1 2 , 0} \mathbf{~ m m}$ \\
\hline
\end{tabular}

29 L'été 2003 est marqué par un bilan hydrique nettement déficitaire avec des valeurs souvent supérieures à $300 \mathrm{~mm}$ (tableau 6), battant celles observées au cours des étés 1976 et 1983. On relève une valeur maximale de $373 \mathrm{~mm}$ à Colmar-Meyenheim (contre 300 et $266 \mathrm{~mm}$ en 1976 et 1983) et de $312 \mathrm{~mm}$ à Entzheim (contre respectivement 305 et $306 \mathrm{~mm}$ ). Le déficit hydrique est un peu moins marqué en Franche-Comté avec des valeurs légèrement supérieures à $250 \mathrm{~mm}$. Par conséquent, les cultures sur les sols ayant une faible réserve utile en eau (cultures de maiis en plaine d'Alsace) ont donc beaucoup souffert du déficit hydrique en 2003.

\section{Pourquoi ce caractère exceptionnel ?}

L'été 2003 survient après une fin d'hiver et un printemps très sec où les conditions anticycloniques prédominent avec une situation de blocage: le flux zonal d'Ouest n'arrive à s'imposer que de manière épisodique. Ces conditions anticycloniques vont perdurer tout au long de l'été, amenant des masses d'air subtropical ou tropical très chaudes issues des latitudes sahariennes. Cette persistance d'advections chaudes subméridiennes ou méridiennes a conduit à des anomalies positives de température (figure 6). 
Figure 6 : Anomalies positives de température observées au cours de l'été 2003 (anomalies calculées à partir de la normale 1961-1990).

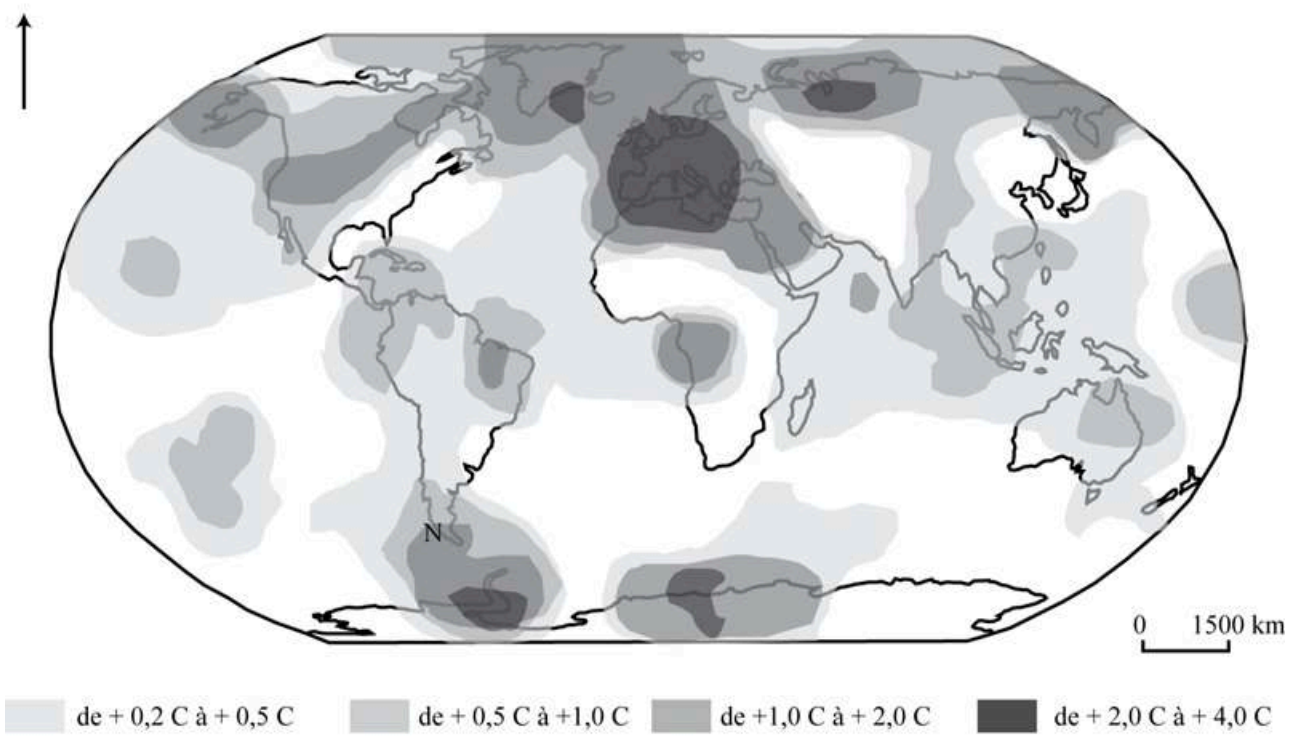

Source : Nasa Goddard Institute for Space Studies. Réalisation: L. Wahl.

Les cartes d'anomalies de température de l'hémisphère Nord indiquent la présence d'une anomalie positive très marquée de +2 à $+4{ }^{\circ} \mathrm{C}$ sur le continent européen pour les trois mois d'été (figure 6). Cette anomalie est particulièrement marquée en juin $\left(+6^{\circ} \mathrm{C}\right.$ à $+7^{\circ} \mathrm{C}$ d'écart par rapport à la normale $\left.1961-1990\right)$ et août $\left(+5^{\circ} \mathrm{C}\right.$ à $\left.+6^{\circ} \mathrm{C}\right)$. Le mois de juillet n'ayant enregistré qu'un excès de $2^{\circ} \mathrm{C}$ à $4^{\circ} \mathrm{C}$. Pour expliquer ces anomalies thermiques, il est nécessaire d'analyser les situations synoptiques qui se sont produites au cours de l'été 2003.

\section{A. Situations synoptiques observées au cours de l'été 2003}

Nous prendrons la classification établie par P. Paul (1995) pour le Nord-Est de la France et le Sud-Ouest de l'Allemagne. L'été 2003 se caractérise par une prédominance de situations anticycloniques ( $61 \%$ des cas), soit un excédent de $15 \%$ par rapport à la normale calculée de 1981 à 2000, contre seulement $39 \%$ de situations perturbées. Cette prédominance de situations anticycloniques permet d'expliquer les durées d'insolation record observées en août et en juin (figure 7). 
Figure 7 : Comparaison des situations synoptiques observées au cours de l'été 2003 avec la moyenne établie sur la période 1981-2000.

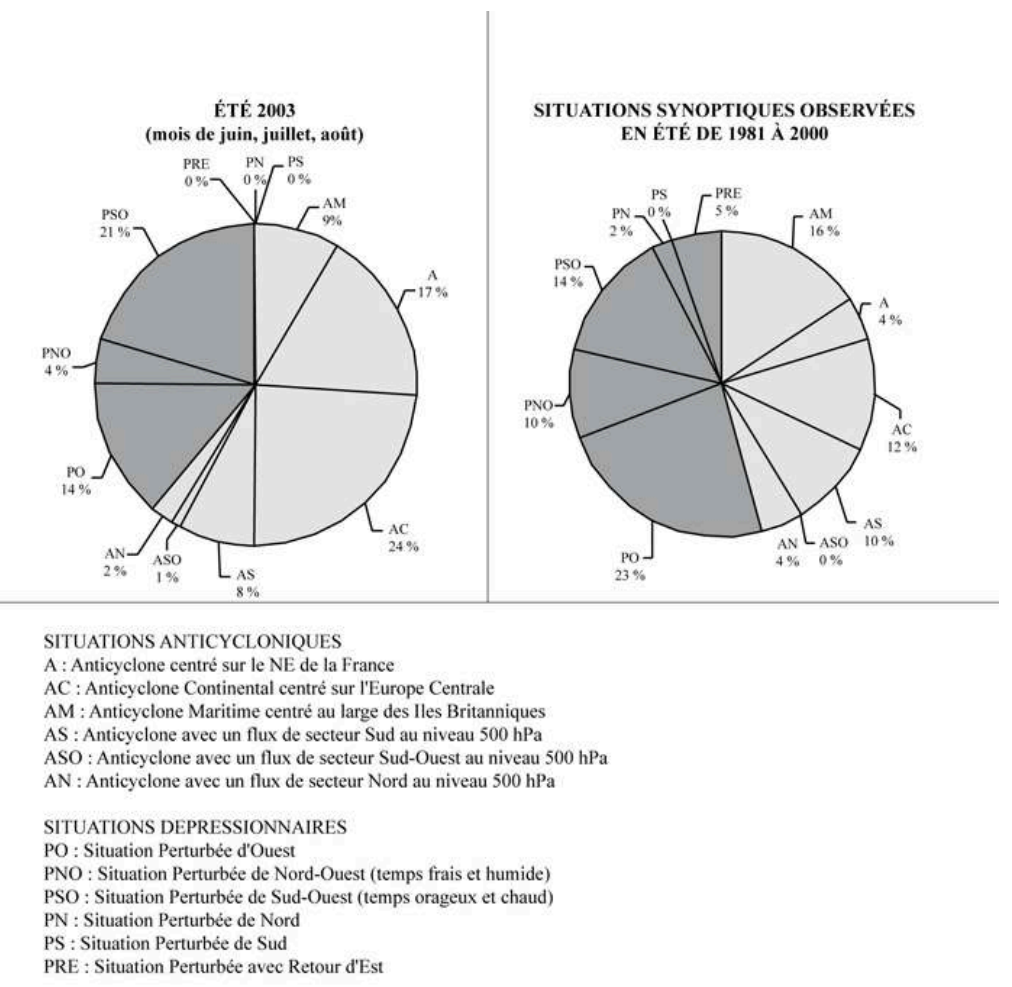

Les situations de type AC (Anticyclone Continental centré sur l'Europe Centrale) représentent près du quart des situations observées $(24 \%)$ et prennent la première place. En général, pour la période allant de 1981 à 2000, ce sont les situations perturbées d'ouest ( $\mathrm{PO})$ qui sont prédominantes avec une fréquence d'apparition de $23 \%$; au cours de l'été 2003, les situations perturbées d'ouest, avec une fréquence de $14 \%$, sont reléguées au $4^{\mathrm{e}}$ rang. Les situations perturbées de sud-ouest (PSO) arrivent en seconde position avec $21 \%$ en 2003 contre seulement $14 \%$. Elles se caractérisent par des températures relativement élevées et un temps devenant lourd et orageux.

À noter l'absence de situations de type PRE (situation Perturbée avec Retour d'Est) et un nombre restreint de situations de type AM (Anticyclone Maritime centré sur la mer du Nord) et PNO (Perturbé de Nord-Ouest) qui présentent respectivement un déficit de $7 \%$ et de $6 \%$. Ces dernières amènent des températures généralement inférieures aux normales saisonnières.

La prédominance des conditions anticycloniques sur l'Europe occidentale a été la cause majeure de l'anomalie thermique de l'été 2003. Les hautes pressions subtropicales se sont étendues fréquemment sur la France et l'Allemagne. Peu marquées en surface à cause de l'effet thermique de l'air très chaud dans les basses couches, une dorsale les reliait parfois à des hautes pressions, établies passagèrement sur la Scandinavie, notamment vers la mi-juillet. La situation en altitude (c'est-à-dire au sein de la moyenne troposphère au niveau de pression $500 \mathrm{hPa}$ ) montre une crête planétaire, constituée d'une masse d'air anormalement chaude et sèche fortement subsidente, qui a contrecarré la plupart des ascendances convectives, génératrices d'orages locaux. Ainsi, le géopotentiel d'août 2003 au-dessus de l'Alsace et de la Lorraine a été supérieur d'environ 100 mètres par rapport à la moyenne du mois d'août, ce qui est exceptionnel. 

atlantiques à proximité du continent européen et a contribué à diriger un flux d'air subtropical très chaud vers la France, en provenance d'Afrique du Nord à travers la péninsule ibérique anticycloniques de type A (17\% contre $4 \%$ ) qui sont caractérisées par une remontée de l'anticyclone des Açores sous la forme d'une dorsale, qui s'accompagne assez fréquemment de remontées d'air très chaud en altitude $\left(+10^{\circ} \mathrm{C}\right.$ à $+15^{\circ} \mathrm{C}$ vers $\left.850 \mathrm{hPa}\right)$. C'est pourquoi, il nous paraît judicieux d'analyser les températures moyennes qui ont été observées à $850 \mathrm{hPa}$.

\section{B. Températures observées à $850 \mathrm{hPa}$}

Si l'on se réfère à la moyenne réalisée à partir de 8 points de mesures (Brest, Paris, Nancy, Strasbourg, Lyon, Genève, Munich et Marseille), l'été 2003 se singularise par un nombre exceptionnellement élevé de journées ( 85 jours pour les 8 postes) où la température a atteint ou dépassé le seuil des $+10^{\circ} \mathrm{C}$ au niveau $850 \mathrm{hPa}$; les valeurs extrêmes s'échelonnent entre 50 jours à Brest et 89 jours à Marseille (figure 8 et tableau 7).

Figure 8 : Températures moyennes journalières observées à $850 \mathrm{hPa}$ à 00 heures UTC pour les étés les plus chauds de la période 1945-2003.

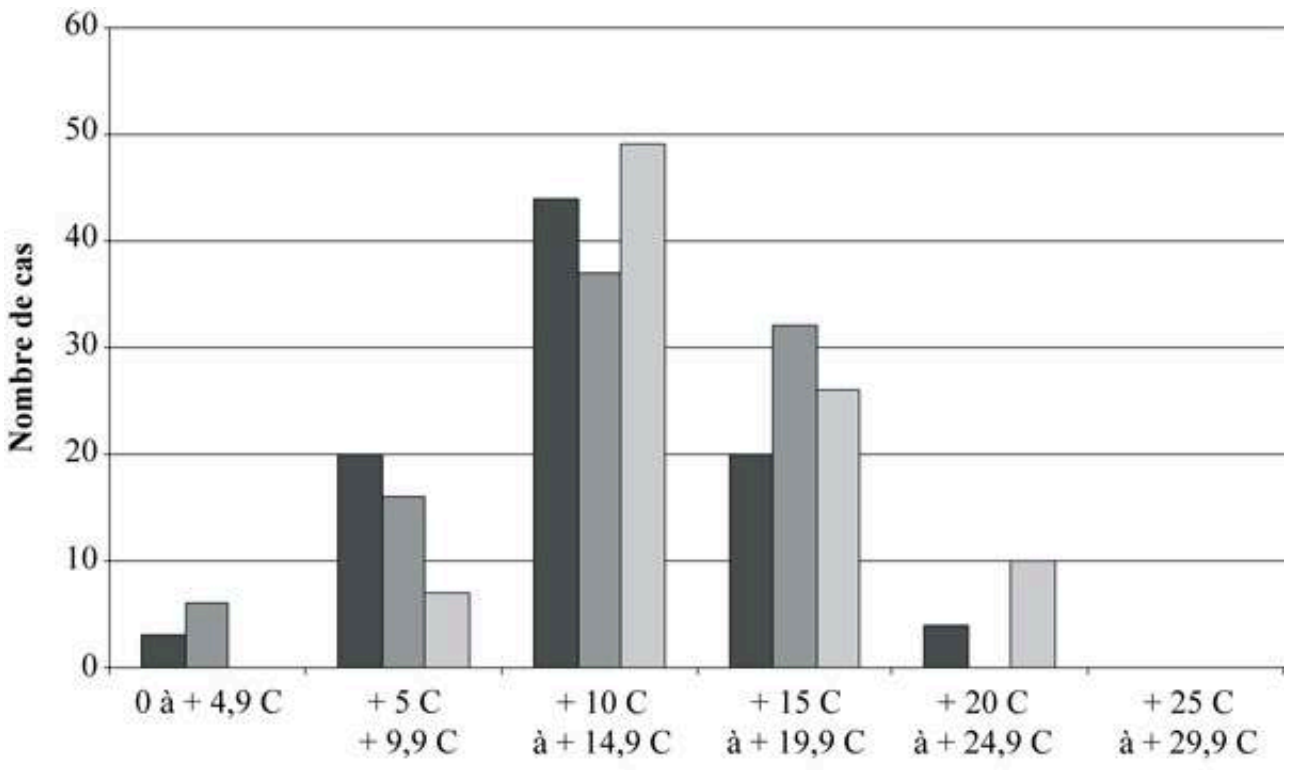

Classes de Tc

ÉTÉ 1983 घ ÉTÉ 1994 घÉTÉ 2003

\begin{tabular}{|l|l|l|l|l|l|l|l|l|}
\hline & Brest & Paris & Nancy & Strasbourg & Munich & Lyon & Genève & Marseille \\
\hline \hline Été 1983 & 12,6 & 11,3 & 12,2 & 10,6 & 12,8 & 13,6 & 13,6 & 15,1 \\
\hline
\end{tabular}




\begin{tabular}{|l|l|l|l|l|l|l|l|l|}
\hline Été 1994 & 12,2 & 10,8 & 11,7 & 10,5 & 12,8 & 13,5 & 13,6 & 15,9 \\
\hline Été 2003 & 11,5 & 13,9 & 12,9 & 14,2 & 14,5 & 16,4 & 16,0 & 18,1 \\
\hline
\end{tabular}

À noter que durant 10 jours consécutifs ( 5 au 14 août), le seuil de $20^{\circ} \mathrm{C}$ a été dépassé, engendrant la forte canicule. Au cours de l'été 1983, qui arrive en seconde position, on ne comptabilise que 68 jours avec $+10^{\circ} \mathrm{C}$ et que 4 jours avec $+20^{\circ} \mathrm{C}$ ! La moyenne des températures enregistrées à $850 \mathrm{hPa}$, calculée pour les 3 mois d'été, s'échelonne entre $+11,5^{\circ} \mathrm{C}$ à Brest, où l'été 1983 a connu une moyenne plus élevée $\left(+12,6^{\circ} \mathrm{C}\right)$, et $+18,1^{\circ} \mathrm{C}$ à Marseille. Outre Marseille, le seuil des $+15^{\circ} \mathrm{C}$ est également dépassé à Lyon et à Genève.

\section{L'épisode de canicule du $1^{\text {er }}$ au 15 août 2003}

Il est exceptionnel, dans la mesure ou l'on a enregistré 14 jours consécutifs avec une température maximale égale ou supérieure à $30^{\circ} \mathrm{C}$, dont 11 avec une température supérieure à $35^{\circ} \mathrm{C}$ dans la plupart des stations. Dans les Alpes Suisses, à l'observatoire météorologique du Jungfraujoch (altitude: $3580 \mathrm{~m}$ ), il ne gèle pas durant 14 jours consécutifs (du $1^{\mathrm{er}}$ au 14 août 2003). Durant cet épisode l'isotherme $0^{\circ} \mathrm{C}$ se situe constamment au-dessus de $4000 \mathrm{~m}$ d'altitude (Bader, 2004).

Cette période de canicule historique s'explique par une remontée de l'anticyclone des Açores qui s'installe durablement sur l'Europe occidentale. Il est doublé en altitude par une remontée d'air très chaud et sec en provenance d'Afrique du Nord. Cet air chaud et sec n'a d'autre effet que de stabiliser la masse d'air empêchant les orages de se déclencher. L'anticyclone "s'auto-entretient " compte tenu d'un sol déjà très sec et d'un gradient de pression très faible. Pour illustrer cela nous prendrons les situations des 8 et 12 août 2003.

La situation du 08 août 2003 (figure 9a) nous indique la présence d'une vaste cellule anticyclonique de $1025 \mathrm{hPa}$ centrée entre l'Ecosse et le Sud de la Norvège, bloquant le flux perturbé au niveau de l'océan Atlantique. Sur le continent bien échauffé, nous avons la présence de «basses pressions thermiques» qui ont pour effet de renforcer l'advection d'air chaud en provenance du Maghreb.

Des développements ponctuels de nuages d'instabilité (cumulo-nimbus) sont observés sur un large quart Nord-Est de la France. L'isotherme $0^{\circ} \mathrm{C}$ se situe aux alentours de 4100 mètres. Les températures maximales sont très élevées avec $+37,2^{\circ} \mathrm{C}$ à Epinal, $+39,3^{\circ} \mathrm{C}$ à Nancy-Essey et $+39,5^{\circ} \mathrm{C}$ à Metz-Frescaty avec des taux d'humidité inférieurs à $30 \%$. Quelques records absolus sont battus au cours de cette journée notamment à Metz-Frescaty $\left(+39,5^{\circ} \mathrm{C}\right)$, Nancy-Essey $\left(+39,3^{\circ} \mathrm{C}\right)$ et Trèves $\left(+39,0^{\circ} \mathrm{C}\right)$.

La situation du 12 août 2003 (figure 9b), montre la présence de deux cellules anticycloniques : l'une centrée sur le Proche-Atlantique de $1024 \mathrm{hPa}$ qui bloque le flux zonal d'Ouest, l'autre de $1023 \mathrm{hPa}$ centrée sur l'Europe centrale, qui amène de l'air très chaud en altitude avec une isotherme $0{ }^{\circ} \mathrm{C}$ située vers 4200 mètres. Les contrastes de pression étant peu marqués avec la présence d'un marais barométrique sur la France les vents sont faibles ce qui favorise l'échauffement des basses couches. C'est au cours de cette journée que l'on observe la plupart des records absolus de chaleur dans le quart Nord-Est de la France (figure 3). 
Figure $9 a$ : 08 août 2003 à 00 heures UTC.

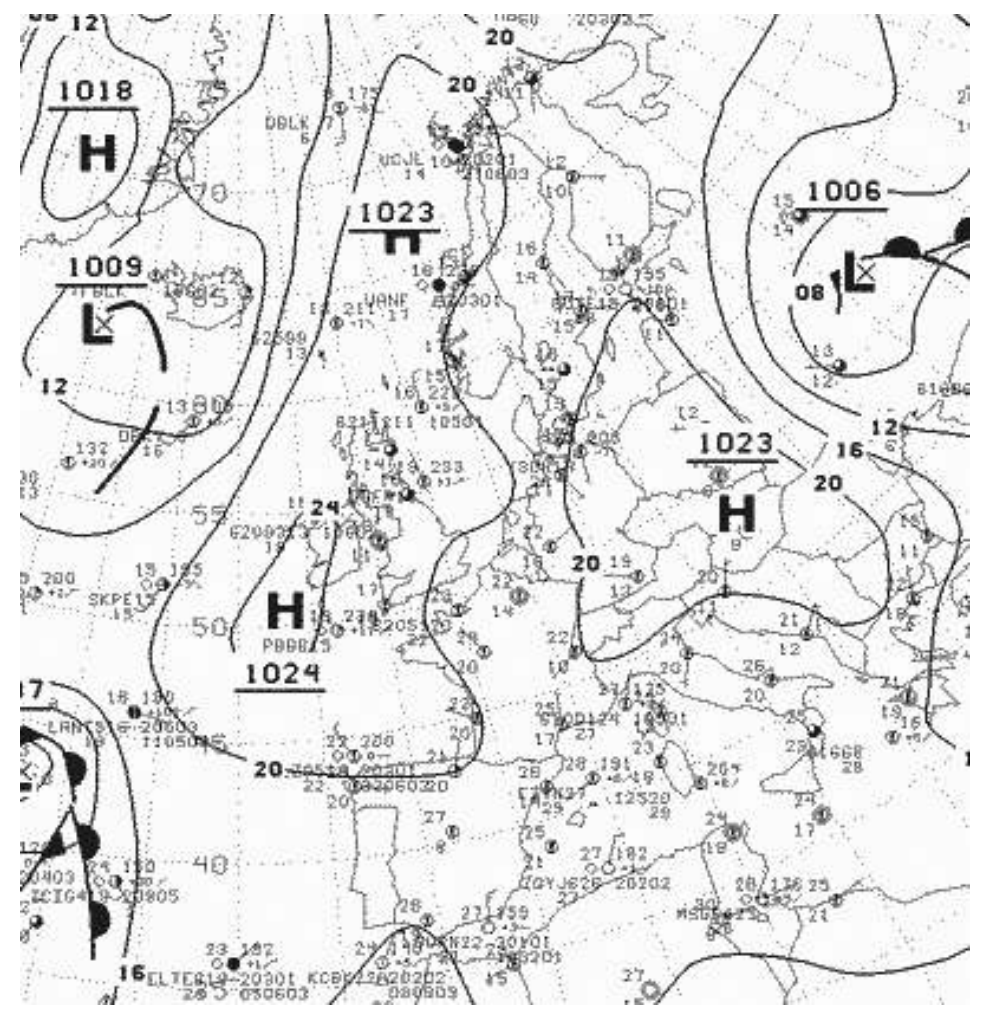

Figure $9 b$ : 12 août 2003 à 00 heures UTC.

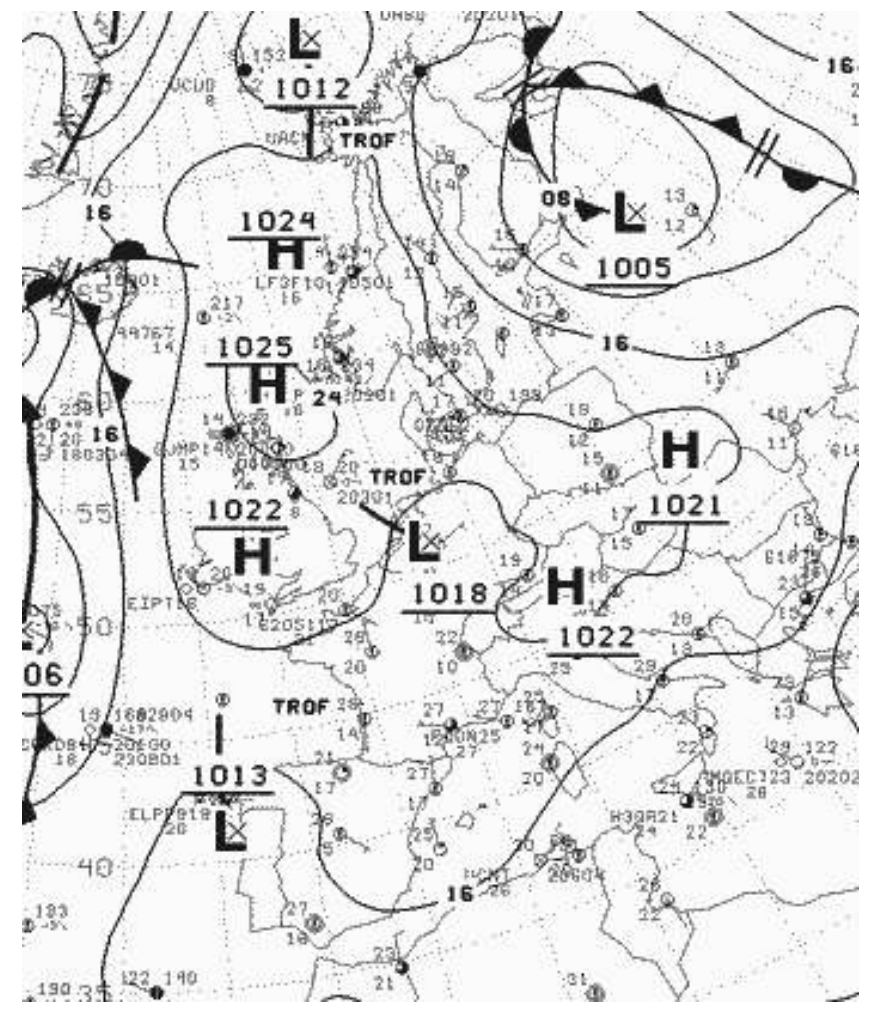




\section{Conclusion}

La chaleur sévère de l'été 2003, exceptionnelle par sa durée et son intensité, est-elle susceptible de se reproduire dans un avenir proche ? Sans doute pas, dans la mesure où la durée probable de retour d'un tel événement, obtenue à l'aide du traitement de données statistiques, est de l'ordre de plusieurs centaines d'années en adoptant l'hypothèse d'une certaine stabilité du climat, celle-ci présentant généralement des variations plus ou moins aléatoires. Il nous paraît en effet difficile d'attribuer cet aléa climatique extrême au seul réchauffement global de la planète, celui-ci ne pouvant fournir au plus qu'un apport supplémentaire de quelques dixièmes de degrés.

Toutefois, il convient d'évoquer que le rapport du GIEC (2001) indique que l'augmentation de la probabilité d'épisodes de fortes chaleurs est simulée par l'ensemble des modèles climatiques à partir de différents scénarios d'augmentation des gaz à effet de serre au cours des prochaines décennies. Avec un scénario moyen d'augmentation de ces gaz, il y aurait une multiplication par un facteur de l'ordre de 5 de la fréquence d'épisodes de fortes chaleurs à la fin du $21^{\mathrm{e}}$ siècle, en prenant comme critère le nombre de jours avec des maxima supérieurs à $35^{\circ} \mathrm{C}$. Si ces projections se confirmaient, les étés torrides deviendraient plutôt la règle que l'exception vers la fin de ce siècle. Les calculs de simulation montrent qu'environ un été sur deux serait alors aussi chaud ou plus que celui de 2003.

Les résultats escomptés des recherches sur l'évolution du climat de la planète et le suivi du climat global au cours des prochaines années devraient permettre de mieux cerner l'évolution du réchauffement global, qui semble s'accélérer depuis une quinzaine d'années.

\section{BIBLIOGRAPHIE}

BADER S. (2004). - « Die extreme Sommerhitze im aussergewöhnlichen Witterungsjahr 2003 », Arbeitsbericht, MétéoSchweiz, Numéro 200, Zürich, 23 p.

BESANÇENOT J.-P. (2003). - « De l'aléa météorologique à la catastrophe sanitaire : la vague de chaleur de l'été 2003 en France », Historiens et Géographes, n³84, p. 395-400.

BRUGGER C.G. (1882). - Beiträge zur Naturchronik der Schweiz, insbesondere der Rhätischen Alpen I, IV Folge, Coire/Chur.

Groupe d'Experts Intergouvernementaux sur l'Évolution du Climat - GIEC (2001). - Bilan 2001 des changements climatiques, les éléments scientifiques, Rapport du groupe de travail 1 du GIEC, $97 \mathrm{p}$.

JANCOVICI J.-M. (2002). - L'avenir climatique. Quel temps ferons-nous ?, Paris, Éditions du Seuil, 288 p. PAUL P. (1995). - Classification des types de temps dans la région du Rhin supérieur, Document interne au CEREG (Centre d'Etudes et de Recherches Eco-Géographiques), Rapport d'activité de l'URA 95, CEREG, Université Louis Pasteur, Strasbourg. 
PAUL P., WAHL L., MORY N., PICHARD C. (2004). - « L'été 2003 en Alsace : une anomalie climatique d'ampleur exceptionnelle », Bulletin de l'Association Philomathique d'Alsace et de Lorraine, $\mathrm{n}^{\circ} 38$, p. 71-78.

PISON G. (2004). - « 60 millions d'habitants en France métropolitaine », Population \& Sociétés, $\mathrm{n}^{\circ}$ 399, Mars, $4 \mathrm{p}$.

PFISTER C. (1999). - Wetternachhersage : 500 Jahre Klimavariationen und Naturkatastrophen, Verlag Paul Haupt, Berne, Stuttgart, Vienne, 340 p.

WAHL L. (2003). - « Analyse du réchauffement climatique contemporain et des types de temps observés dans le Nord-Est de la France et le Sud-Ouest de l'Allemagne de 1981 à 2000 ", Publications de l'Association Internationale de Climatologie (AIC), Vol. n 15, p. 441-448.

\section{RÉSUMÉS}

L'été 2003 restera dans les annales climatiques comme un événement météorologique exceptionnel de par son ampleur et sa durée. 22 postes synoptiques situés dans le quart Nord-Est de la France, ont été sélectionnés pour faire ressortir les principales caractéristiques de cet été. Plusieurs paramètres (températures, précipitations, insolation) ont été comparés aux normales climatiques de la période 1971-2000. Au vu des écarts thermiques observés, trois périodes de canicule ont pu être mises en évidence. Elles sont d'ampleur inégale selon la latitude et la région considérées. Une analyse détaillée des conditions synoptiques a été réalisée afin de connaitre les facteurs à l'origine de la canicule. L'épisode du 3 au 14 août 2003 a fait l'objet d'une attention particulière.

Owing to its magnitude and duration, the summer of 2003 will be remembered as an outstanding meteorological event. 22 synoptical weather stations located in northeastern France have been selected in view of analysing the main features of this summer. Three meteorological parameters (air temperature, rainfall, sunshine duration) have been compared to their 1971-2000 climate normals. Air temperature anomalies allow identifying three heat waves during summer 2003 with different magnitudes according to the latitude and the considered region. A detailed analysis of synoptical weather conditions has been performed in order to identify heat wave explanatory factors, especially for the $3^{\text {rd }}$ to $14^{\text {th }}$ of August 2003 episod.

Der Sommer 2003 wird in den Klimatologischen Annalen als einem besonderen météorologische Ereignis, an Intensität und Dauer, bezeichnet. Mit Hilfe, 22 synoptische Klimastationen im NordOsten von Frankreich, wurden die hauptsächlichen Charakteristiken dieses Sommers analysiert. Mehrere Parameter (Temperatur, Niederschlag, Sonnenscheindauer) wurden mit den Normalen vom Zeitraum 1971-2000 verglichen. In Betrachtung der verschiedenen Abweichungen, wurden drei Hitzewellen deutlich Identifiziert. Von dem Breitengrad und der Regionen abgesehen, sind sie von verschiedener Intensität. Mit Hilfe von synoptischen Daten und Karten wurden die Ursachen der Hitzewelle analysiert. Der Zeitraum vom 3. bis 14. August wurde aufmerksam untersucht. 
INDEX

Mots-clés : canicule, été 2003, insolation, jours de chaleur, Nord-Est de la France, précipitations, températures

Schlüsselwörter : Hitzewelle, Niederschlag, Nordostfrankreich, Sommer 2003, Sommertage, Sonnenscheindauer, Temperatur

Keywords : heat wave, Northeastern France, rainfall, summer 2003, summerday, sunshine, temperatures

\section{AUTEURS}

LAURENT WAHL

Département de Géographie, Université de Nancy 2, 23 bd Albert I ${ }^{\mathrm{er}}$, BP 3397, F-54015 Nancy

Cedex

\section{PATRICE PAUL}

Faculté de Géographie, Université Louis Pasteur, 3 rue de l'Argonne, F-67083 Strasbourg Cedex

\section{CLAUDE PICHARD}

8 rue des Ormes, F-67118 Geispolsheim

\section{NICOLAS MORY}

24, avenue André Malraux, F-67400 Illkirch

\section{GILLES DROGUE}

Cellule de Recherche en Environnement et Biotechnologies, Université de Luxembourg, 162a, avenue de la Faiencerie, L-1511 Luxembourg, LUXEMBOURG

\section{LAURENT PFISTER}

Cellule de Recherche en Environnement et Biotechnologies, Université de Luxembourg, 162a, avenue de la Faiencerie, L-1511 Luxembourg, LUXEMBOURG 